\title{
Transrepression of the estrogen
} receptor promoter by calcitriol in human breast cancer cells via two negative vitamin $D$ response elements

\section{Srilatha Swami, Aruna V Krishnan, Lihong Peng, Johan Lundqvist ${ }^{1}$ and David Feldman}

Division of Endocrinology, Department of Medicine, Stanford University School of Medicine, 300 Pasteur Drive, Room S025, Stanford, California 94305-5103, USA

'Breast Cancer Group, Cell Death and Metabolism, Danish Cancer Society Research Center, Strandboulevarden 49, DK-2100 Copenhagen $\varnothing$, Denmark
Correspondence should be addressed to D Feldman

Email

dfeldman@stanford.edu

\begin{abstract}
Calcitriol (1,25-dihydroxyvitamin $D_{3}$ ), the hormonally active metabolite of vitamin $D$, exerts its anti-proliferative activity in breast cancer $(\mathrm{BCa})$ cells by multiple mechanisms including the downregulation of the expression of estrogen receptor $\alpha$ (ER). We analyzed an $\sim 3.5 \mathrm{~kb} E R$ promoter sequence and demonstrated the presence of two potential negative vitamin D response elements ( $n V D R E s$ ), a newly identified putative nVDRE upstream at -2488 to $-2473 \mathrm{bp}$ (distal nVDRE) and a previously published sequence (proximal nVDRE) at -94 to $-70 \mathrm{bp}$ proximal to the P1 start site. Transactivation analysis using $E R$ promoter deletion constructs and heterologous promoter-reporter constructs revealed that both nVDREs functioned to mediate calcitriol transrepression. In the electrophoretic mobility shift assay, the vitamin D receptor (VDR) showed strong binding to both nVDREs in the presence of calcitriol, and the chromatin immunoprecipitation assay demonstrated the recruitment of the VDR to the distal nVDRE site. Mutations in the $5^{\prime}$ hexameric DNA sequence of the distal nVDRE resulted in the loss of calcitriol-mediated transrepression and the inhibition of protein-DNA complex formation, demonstrating the importance of these nucleotides in VDR DNA binding and transrepression. A putative nuclear factor-Y (NFY) binding site, identified within the distal nVDRE, led to the findings that NFY bound to the distal nVDRE site interfered with the binding of the VDR at the site and reduced calcitriolmediated transrepression. In conclusion, the ER promoter region contains two negative VDREs that act in concert to bind to the VDR and both nVDREs are required for the maximal inhibition of $E R$ expression by calcitriol. The suppression of $E R$ expression and estrogen-mediated signaling by calcitriol in BCa cells suggests that vitamin D may be useful in the treatment of ER+ BCa.
\end{abstract}

\footnotetext{
Key Words

- vitamin D

- calcitriol

- transrepression

- estrogen receptor

- nVDREs

- estrogen signaling

- breast cancer cells
}

Endocrine-Related Cancer (2013) 20, 565-577
(C) 2013 Society for Endocrinology Printed in Great Britain
Published by Bioscientifica Ltd 


\section{Introduction}

Breast cancer $(\mathrm{BCa})$ is the most common cancer in women in the USA (Siegel et al. 2012). Estrogens drive the proliferation of mammary epithelial cells and therefore promote the growth of estrogen receptor-positive $(\mathrm{ER}+)$ BCa cells. Estrogens act by binding to the estrogen receptor $\alpha$ (ER) to regulate gene expression. Therapy for $\mathrm{ER}+\mathrm{BCa}$, therefore, includes hormonal treatments such as those with aromatase inhibitors that inhibit estrogen synthesis and selective ER modulators that block estrogen action in the breast by binding to the $E R$ and acting as antagonists (Burstein et al. 2010, Tomao et al. 2011). Another approach to inhibit the growth of ER+ BCa is to decrease the levels of $\mathrm{ER}$, the nuclear receptor that mediates the growth-promoting effects of estrogens in breast epithelial cells using compounds that act as selective ER downregulators such as fulvestrant (McDonnell \& Wardell 2010, Nilsson et al. 2011).

Calcitriol, the hormonally active form of vitamin D (1,25-dihydroxyvitamin $\left.\mathrm{D}_{3}\right)$, plays an important role in calcium homeostasis through its actions (Feldman et al. 2013). In addition to these classical actions, calcitriol also exhibits anti-proliferative, pro-differentiating, and antiinflammatory activities, indicating its potential use in the prevention and treatment of multiple cancers including BCa (Beer \& Myrthue 2004, Deeb et al. 2007, Krishnan \& Feldman 2011, Pereira et al. 2012, Welsh 2012). Interestingly, our studies reveal that in ER + BCa cells calcitriol inhibits both estrogen synthesis and signaling, leading to a significant attenuation of estrogen stimulation of cell growth (Krishnan et al. 2010a). We have shown that calcitriol acts as a selective aromatase modulator (SAM) and decreases estrogen synthesis in BCa cells and the surrounding mammary adipose tissue by suppressing aromatase expression (Krishnan et al. 2010b, Swami et al. 2011). Furthermore, calcitriol and its analogs also downregulate ER expression in BCa cells (James et al. 1994, Simboli-Campbell et al. 1997, Swami et al. 2000), thereby causing a significant inhibition of estrogenic bioresponses in these cells, including the suppression of estrogenstimulated cell growth (Demirpence et al. 1994, Swami et al. 2000).

Our earlier study (Swami et al. 2000) and that of Stoica et al. (1999) revealed that the negative regulation of $E R$ expression by calcitriol occurred at the transcriptional level. This transcriptional repression is probably mediated through the binding of the vitamin D receptor (VDR) to one or more negative vitamin $\mathrm{D}$ response elements (nVDREs) present in the ER promoter. The current study explores the mechanism of the transcriptional repression of the $E R$ gene by calcitriol. Recent advances in the genomewide analysis of transcriptional regulation by calcitriol have revealed that the VDREs could be located both proximal and distal to the transcriptional start sites of target genes and many, in fact, are located in clusters hundreds of kilobases from their target genes (Pike \& Meyer 2010, Pike 2011, Pike et al. 2011a). However, our current analysis demonstrates that the functional activities of two nVDRE sites within an $\sim 3.5 \mathrm{~kb}$ promoter region of the $E R$ gene recapitulate the magnitude of the downregulation of ER expression observed in BCa cells following calcitriol treatment. One of these sites, the proximal nVDRE identified previously by Stoica et al. (1999), is an imperfect palindromic sequence located at -94 to $-70 \mathrm{bp}$ of the ER gene with reference to the P1 start site, the major start site in ER + BCa cells (deConinck et al. 1995). Herein, we report the identification and characterization of another putative nVDRE (distal nVDRE) further upstream of the published proximal site that also contributes to the calcitriol-mediated transrepression of $E R$.

\section{Materials and methods}

\section{Materials}

The human BCa cell lines used in the study were obtained from the American Type Culture Collection (Rockville, MD, USA). Tissue culture media, supplements, and FCS were obtained from Gibco BRL, Lonza (Walkersville, MD, USA), and Mediatech, Inc. (Herndon, VA, USA). All antibodies were obtained from Santa Cruz Biotechnology. All other chemicals and reagents were procured from Sigma Chemical Co., unless otherwise indicated.

\section{Methods}

Cell culture The human BCa cell lines MCF-7, ZR75-1, and T47D were cultured at $37{ }^{\circ} \mathrm{C}$ under $5 \% \quad \mathrm{CO}_{2}$ atmosphere using RPMI-1640 medium supplemented with $10 \%$ calf serum, penicillin $(100 \mathrm{U} / \mathrm{ml})$, and streptomycin $(100 \mu \mathrm{g} / \mathrm{ml})$.

Plasmids $E R$ promoter-reporter plasmids containing a series of $5^{\prime}$ deletions with reference to the P1 start site were a kind gift from Dr Ronald Weigel (formerly at the Stanford University and currently at the University of Iowa, Iowa City, IA, USA). The construction

Published by Bioscientifica Ltd. 
of these plasmids has been described previously (deConinck et al. 1995). The NFYA expression plasmids were a kind gift from Dr Roberto Mantovani (Universita di Milano, Milano, Italy).

Heterologous constructs for VDRE analysis We refer to the previously described VDRE by Stoica et al. (1999) ( -94 to -70 ) as the proximal nVDRE and the newly discovered upstream site as the distal nVDRE $(-2488$ to -2473$)$. A 230 bp KpnI-HindIII fragment (+ $230 / 0)$ from the plasmid pGL2-ER promoter $(-3500$ to +230) was cloned into the KpnI/SmaI sites of the heterologous Simian virus 40 (SV40) promoter-driven luciferase reporter vector pGL3 (Promega) to generate the nVDRE(null) construct. Oligonucleotides enclosing the proximal nVDRE (31 nucleotides), the distal nVDRE (27 nucleotides), and an oligonucleotide containing both nVDRE sequences (58 nucleotides) containing a MluI overhang at the $5^{\prime}$ end were synthesized (Operon Technologies, Alameda, CA, USA). Single copies of each of these oligonucleotides were annealed and ligated to the MluI site of the nVDRE(null) plasmid to generate the heterologous constructs containing the proximal, distal, or both nVDRE sequences. The orientation of each insert in the heterologous constructs was verified by sequencing.

\section{Mutagenesis of the distal nVDRE sequence} in the natural and heterologous $E R$ promoter constructs Mutations in the $5^{\prime}$ half-site (potential RXR binding site) and the $3^{\prime}$ half-site (potential VDR binding site) in the distal nVDRE sequence were introduced into the $-3500 /+230 /$ pGL2-Basic reporter construct using the GeneEditor site-directed mutagenesis system (Promega). To generate the heterologous mutant constructs, two pairs of oligonucleotides containing the same mutations in the distal nVDRE sequence with the MluI site overhangs were synthesized and cloned into the nVDRE(null) plasmid. Positive clones identified by sequencing were used in the transactivation assays.

Transient transfections and luciferase assays The various $E R$ promoter-luciferase reporter constructs were transiently transfected into the ZR75-1 or MCF-7 cells using LipofectAmine (Life Technologies/Invitrogen). A renilla luciferase plasmid (pRLnull, Promega) was co-transfected to control for transfection efficiency. Following transfections, the cells were treated with a vehicle ( $0.1 \%$ ethanol) or $100 \mathrm{nM}$ calcitriol for $24 \mathrm{~h}$. Reporter and renilla luciferase activities were measured using the Dual Luciferase Assay Kit (Promega) (Peng et al. 2004).
Electrophoretic mobility shift assay Electrophoretic mobility shift assay (EMSA), as described previously (Peng et al. 2004), was used to analyze the binding of the VDR to the putative nVDREs in the ER promoter using complementary oligonucleotides for the distal and proximal nVDREs. ZR75-1 cell extracts were incubated for $15-20 \mathrm{~min}$ with $100 \mathrm{nM}$ calcitriol and $\left[{ }^{32} \mathrm{P}\right]$-labeled oligonucleotide probes. For competition assays, a 500-fold molar excess of the corresponding unlabeled oligonucleotides was added to the reaction mixture before the addition of the labeled probes. In some reactions, $2 \mu \mathrm{g}$ of an anti-VDR polyclonal antibody (H-81) or an anti-RXR $\alpha$ polyclonal antibody (D20) were added to the reaction mixture $40 \mathrm{~min}$ prior to the addition of the labeled probe. After additional incubation for $20 \mathrm{~min}$, the DNA-protein complexes were separated by electrophoresis on 5\% nondenaturing polyacrylamide gels in a Tris-borate-EDTA buffer and visualized by autoradiography (Peng et al. 2004).

Chromatin immunoprecipitation assay $Z R 75-1$ and MCF-7 cells treated with a vehicle or $100 \mathrm{nM}$ calcitriol for $2 \mathrm{~h}$ (for VDR) or $24 \mathrm{~h}$ (for RXR $\alpha$ and NFYA) were subjected to a chromatin immunoprecipitation (ChIP) analysis using the EZ-ChIP kit (Upstate/EMD Millipore Corp., Billerica, MA, USA) as described previously (Peng et al. 2004). Immunoprecipitation of the chromatin fragments was carried out with IgG or a mixture of polyclonal antibodies against the VDR (N-20, C-20, and $\mathrm{H}-81$ ), a polyclonal antibody against $\mathrm{RXR} \alpha$ (D-20), or a polyclonal antibody against NFYA (C-18). After washing and reverse cross-linking, qPCR was performed on the eluted DNA using primers designed to amplify a $178 \mathrm{bp} E R \alpha$ promoter fragment from -2581 to $-2403 \mathrm{bp}$ encompassing the distal nVDRE: forward, 5'-GTGGGTTTGGTTAACGAAGTG -3'; reverse, 5'-CCTTCTGGGATACATGTGGATCA-3'. QPCR data are expressed as the ratio of the amount of total immunoprecipitated DNA (bound) to the amount of input DNA. The PCR products were also visualized by agarose gel electrophoresis and ethidium bromide staining.

Western blot analysis Nuclear extracts of the BCa cells were subjected to $10 \%$ SDS-PAGE, transferred onto nitrocellulose membranes, and immunoblotted with an anti-mouse MAB to human ER (F-10; 1:500 dilution in 1\% Carnation nonfat milk) as described previously (Swami et al. 2000). The blots were then probed with a HRPconjugated anti-mouse secondary antibody, and the immunoreactive bands were detected using an ECL Kit (Amersham).

Published by Bioscientifica Ltd. 


\section{Statistical analysis}

All values are presented as means \pm s.E.M. Data were evaluated by ANOVA with Scheffe's test as the post hoc analysis or Student's $t$-test where appropriate using the GraphPad Prism 5 Software (GraphPad Software, Inc., La Jolla, CA, USA).

\section{Results}

\section{Suppression of $E R$ promoter activity by calcitriol in BCa cell lines}

The plasmid containing the full-length $E R$ promoter sequence $(-3500$ to +230$)$ cloned into the pGL2-Basic vector was transiently transfected into various BCa cell lines along with a VDR expression plasmid (pSG5-VDR) and a renilla luciferase plasmid. Treatment of the cells with $100 \mathrm{nM}$ calcitriol for $24 \mathrm{~h}$ resulted in significant decreases $(\sim 65-80 \%)$ in relative luciferase activity (Fig. 1A), demonstrating a substantial suppression of $E R$ promoter activity by calcitriol in the various cell lines. In the ZR75-1 cells, calcitriol treatment decreased reporter activity in a dose-dependent manner (Fig. 1B). These data support our hypothesis that calcitriol transrepresses $E R \alpha$ transcription, probably acting through one or more nVDREs present in the ER promoter. Stoica et al. (1999) identified an imperfect palindromic sequence at -94 to $-70 \mathrm{bp}$ of the ER promoter with reference to the P1 start site and demonstrated that it acted as a nVDRE in reporter assays. Upon further in silico analysis, we have found another putative nVDRE upstream at -2488 to $-2473 \mathrm{bp}$ with reference to the $\mathrm{P} 1$ start site, hereinafter referred to as the distal nVDRE (Fig. 2A).

\section{Deletion analysis of the $E R$ promoter}

To further define the nVDREs in the 3500 bp ER promoter fragment, deletion constructs of the promoter cloned into the pGL2-Basic vector were transiently transfected into the ZR75-1 cells and calcitriol suppression of reporter activity was determined. As shown in Fig. 2A, calcitriol treatment significantly inhibited the reporter activity of the fulllength $E R$ promoter-luc $(-3500 /+230$ LUC), causing an $\sim 72 \%$ decrease $(P<0.001)$. The removal of the $5^{\prime}$ sequence from -3500 to -701 containing the distal nVDRE $(-700 /+230$ LUC) significantly reduced the magnitude of calcitriol-mediated suppression when compared with the full-length promoter ( $\sim 54$ vs $\sim 72 \%$ decrease, $P<0.05)$, suggesting that the distal nVDRE sequence mediates a substantial portion of the negative regulation of the $E R$ promoter by calcitriol. Interestingly, the removal of the -3500 to -701 fragment increased the basal reporter activity compared with the full-length promoter ( $\sim 2 \%$ increase, $P<0.05$ ), suggesting that this region may contain binding sites for other negative regulators
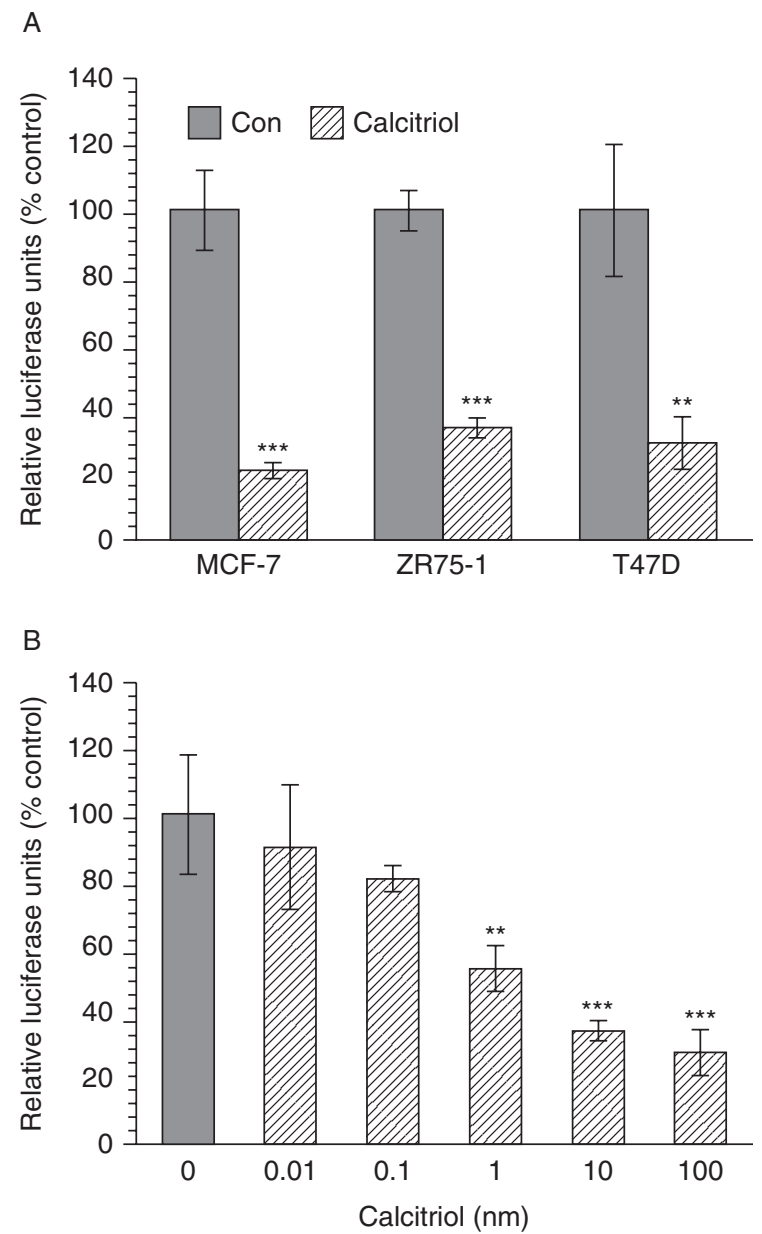

Figure 1

Transcriptional repression of the human $E R$ promoter by calcitriol in breast cancer cell lines. (A) The human $E R$ promoter-luciferase construct ( $\sim 3.5 \mathrm{~kb}$ ) was transiently transfected along with a VDR expression vector (pSG5-VDR) into the MCF-7, ZR75-1, and T47D human breast cancer cell lines. A renilla luciferase expression vector was co-transfected to control for transfection efficiency. The cells were treated with either a vehicle (Con) or $100 \mathrm{nM}$ calcitriol for $24 \mathrm{~h}$ and the luciferase activity was assayed. Relative luciferase activity in the calcitriol-treated cells was compared with that of the vehicletreated controls in each transfection that was set at $100 \%$. Values represent means \pm S.E.M. of at least four independent transfections performed in triplicate. ${ }^{* *} P<0.01$ and $* * * P<0.001$ when compared with the control. (B) Dose response of the ER promoter to calcitriol in the ZR75-1 human breast cancer cells. ZR75-1 cells transiently transfected with the human $E R$ promoter, as described above, were treated with either a vehicle (Con) or various concentrations of calcitriol (B) for $24 \mathrm{~h}$. Relative luciferase activity in the calcitriol-treated cells was compared with that of the vehicle-treated controls in each transfection that was set at $100 \%$. Values represent means \pm S.E.M. of at least four independent transfections performed in triplicate. ${ }^{* *} P<0.01$ and $* * * P<0.001$ when compared with the control.

Published by Bioscientifica Ltd. 
A

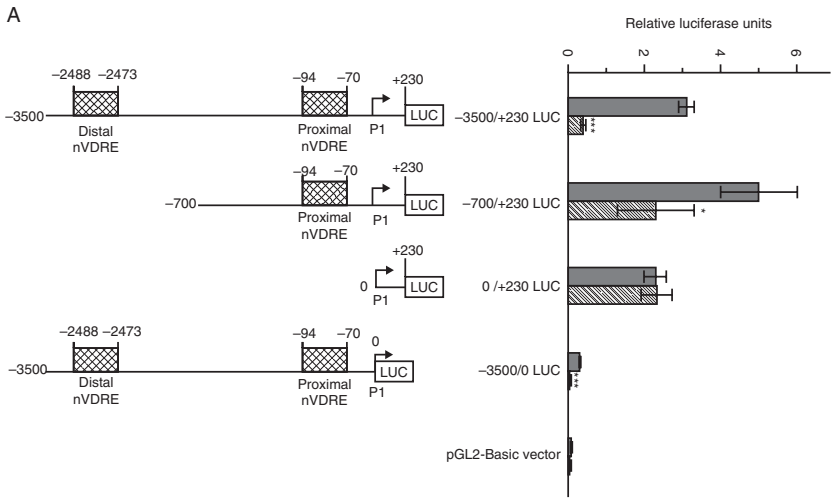

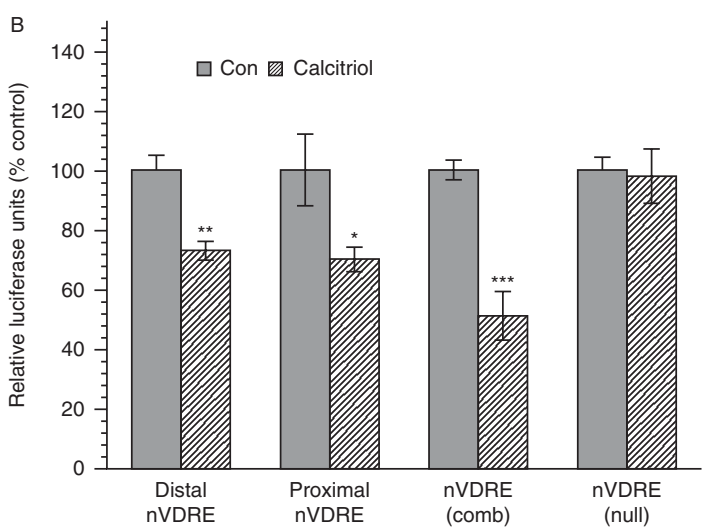

Figure 2

Transcriptional activity of $E R$ promoter constructs. (A) Diagrammatic representation of the $E R$ promoter $(\sim 3.5 \mathrm{~kb})$ and the various deletions constructs cloned into the pGL2-Basic reporter vector (left panel) with reference to the location of the distal and proximal nVDRE sites. The numbers are relative to the $\mathrm{P} 1$ transcription start site that is set at 0 . The constructs were transiently transfected into the ZR75-1 cells as described in the Subjects and methods section and treated with a vehicle (Con) or $100 \mathrm{nM}$ calcitriol for $24 \mathrm{~h}$ (right panel). The values are expressed as a ratio of the $E R$-luc normalized to renilla-luc activity in order to correct for transfection efficiency. Values represent means \pm S.E.M. of at least four independent transfections performed in triplicate. ${ }^{*} P<0.05$ and $* * * P<0.01$ when compared with the control. (B) Reporter activity of the

of $E R$ promoter activity. Deletion of the entire $5^{\prime}$ promoter region $(0 /+230$ LUC) eliminating both nVDREs completely abolished the calcitriol-mediated suppression of reporter activity, demonstrating that both distal and proximal nVDREs are necessary for the full suppressive effect of calcitriol. Deletion of a $230 \mathrm{bp}$ fragment from the P1 start site containing the $5^{\prime}$ untranslated leader of the ER gene ( $-3500 / 0$ LUC) greatly reduced ( $~ 90 \%$ decrease, $P<0.01)$ the basal promoter activity when compared with the basal activity of the full-length $3500 \mathrm{bp}$ promoter including the $230 \mathrm{bp}$ fragment, indicating that this $230 \mathrm{bp}$ region contained a powerful simulator of $E R$ expression. However, even with the reduction in the basal promoter activity of this deletion construct, significant calcitriolmediated further suppression was still evident ( $~ 85 \%$ decrease in calcitriol-treated cells vs basal activity of the $-3500 / 0$ construct, $P<0.001)$.

\section{Conferment of calcitriol responsiveness to a heterologous promoter by ER nVDREs}

To further demonstrate that the two nVDRE sequences specifically respond to calcitriol, heterologous constructs containing a single copy each of the proximal and distal nVDRE sequences or both VDRE sequences were transiently transfected into the ZR75-1 cells along with heterologous constructs of the distal and proximal nVDREs. Single copies of the wild-type distal or proximal or both $E R \mathrm{nVDREs}$ in the sense direction were cloned into the pGL3-promoter-reporter vector and transfected into the ZR75-1 cells as described in the Subjects and methods section. The transfected cells were treated with either a vehicle or $100 \mathrm{nM}$ calcitriol for $24 \mathrm{~h}$. A null reporter containing a nonsense sequence was used as a negative control to assess the effects of calcitriol on the heterologous constructs. Relative luciferase activity in the calcitriol-treated cells was compared with that of the vehicle-treated controls in each transfection that was set at $100 \%$. Values represent means \pm s.E.M. of at least four independent transfections performed in triplicate. ${ }^{*} P<0.05, * * P<0.01$, and $* * * P<0.001$ when compared with the control.

pSG5-VDR and the renilla luciferase plasmid and reporter activity was assessed. Treatment with $100 \mathrm{nM}$ calcitriol caused an $\sim 25 \%$ decrease in reporter activity in cells transfected with a construct containing either the distal nVDRE or the proximal nVDRE alone (Fig. 2B). In cells transfected with a construct containing both the nVDREs (nVDRE(comb)), calcitriol-induced suppression of reporter activity was higher compared with the constructs with just either the proximal or distal nVDRE ( $\sim 49 \%$ decrease, $P<0.05)$. In ZR75-1 cells transfected with the pGL3 promoter vector lacking any VDRE sequence (nVDRE(null)), calcitriol did not suppress reporter activity (Fig. 2B).

\section{Mutational analysis of the nVDRE sequences}

A mutational analysis was carried out to confirm that the suppression of the ER promoter by calcitriol was mediated through the distal nVDRE sequence present in the $E R$ promoter (Fig. 3A and B). Stoica et al. (1999) demonstrated that when the proximal nVDRE sequence was mutated to give rise to a nonsense sequence inserted $5^{\prime}$ of a SV40 promoter-chloramphenicol acetyl transferase reporter plasmid, calcitriol suppression of reporter activity was lost following transfection of this heterologous promoter construct into MCF-7 cells. In the current study, we used site-directed mutagenesis to create point mutations in the

Published by Bioscientifica Ltd. 
distal nVDRE. We generated mutants with base changes in both the $3^{\prime}$ half-site ( $3^{\prime}$ MUT) and $5^{\prime}$ half-site ( $5^{\prime}$ MUT) of the distal nVDRE (Fig. 3C). The mutations were created both in the native promoter context $(-3500$ to $+230 E R$ promoterluc) and in the heterologous construct in the pGL3 vector. Various wild-type (WT) and mutant constructs were transfected into the ZR75-1 cells and calcitriol responsive-

A ER promoter

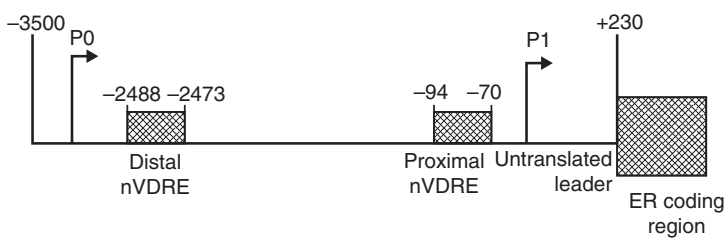

B

nVDRE Sequences

Distal nVDRE GTGCAA TCG GAGGCA

Proximal nVDRE CAGGCAaggcaacagtcccTGGCCG

C Mutations in distal nVDRE

Wild-type (WT) GTGCAA TCG GAGGCA

$3^{\prime}$ mutatiom ( $3^{\prime} \mathrm{MUT}$ ) GTGCAA TCG $\mathrm{taGeCA}$

$5^{\prime}$ mutatiom (5'MUT) t $\mathrm{T}$ tCgA TCG GAGGCA

D Full promoter constructs

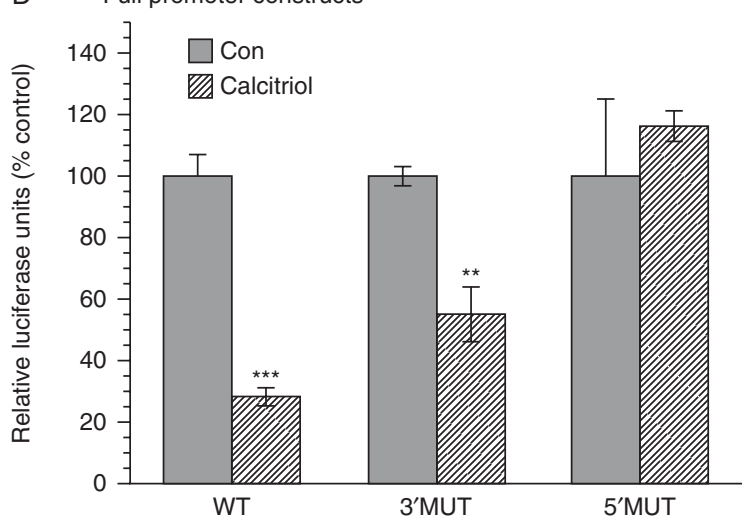

E Heterologous constructs

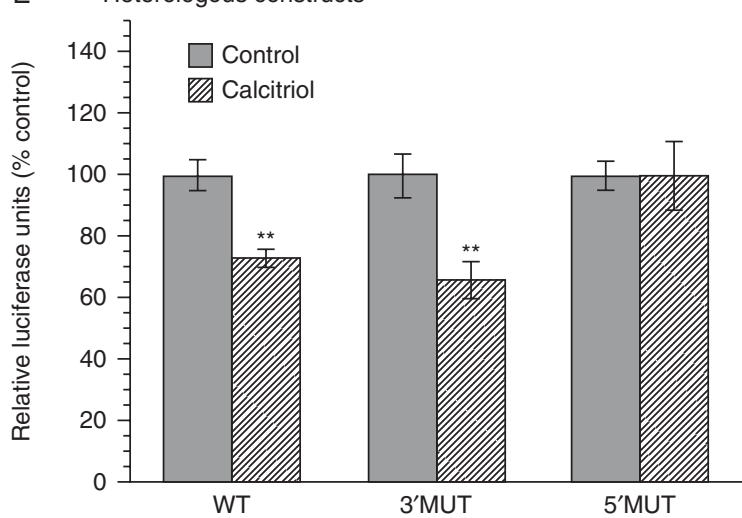

http://erc.endocrinology-journals.org DOI: $10.1530 /$ ERC-12-0281 ness was assessed by measuring reporter activity. As shown in Fig. 3D, calcitriol treatment caused a significant suppression of activity in the native WT promoter construct as well as in the $3^{\prime}$ MUT construct. Mutations in the $5^{\prime}$ half-site of the distal nVDRE ( $5^{\prime}$ MUT) in the full promoter construct resulted in an approximately fivefold decrease in the basal promoter activity. Furthermore, a loss of calcitriol-mediated suppression of promoter activity was also observed with the $5^{\prime}$ MUT construct (Fig. 3D). Similar results were observed when the same mutations were created in the distal nVDRE in the heterologous construct (Fig. 3E). Mutations in the $5^{\prime}$ half-site but not in the $3^{\prime}$ halfsite abolished the suppressive effect of calcitriol, suggesting that the $5^{\prime}$ hexameric sequence is required for calcitriol suppressor activity via the distal nVDRE (Fig. 3E).

\section{Analysis of the binding of the VDR to the nVDREs by EMSA}

To demonstrate the binding of the VDR to the nVDREs in the ER promoter, we carried out EMSA (Fig. 4). In this assay, we used extracts of ZR75-1 cells that contained endogenous RXR and VDR as well as the VDR expressed by the vector pSG5-VDR that was transfected into the cells. Lane 1 in Fig. 4A and B represents the free probe. As shown in Fig. 4A, when incubated with the radiolabeled distal nVDRE probe, the formation of a protein-DNA complex was detected (Fig. 4A, lane 2). The addition of calcitriol substantially increased the intensity of this complex (Fig. 4A, lane 3). Competition with an unlabeled distal nVDRE oligonucleotide showed a decrease in the band intensity of the complex (Fig. 4A, lane 4). The addition of the anti-VDR antibodies clearly inhibited complex formation (Fig. 4A, lane 5). Similarly, the addition of the anti-RXR $\alpha$ antibodies also inhibited complex formation (Fig. 4A, lane 6). However, with both antibodies, the presence of lower-molecular-

Figure 3

Distal nVDRE sequences and mutations. A schematic representation of the $E R$ promoter with the location of the two putative nVDREs (A) and their sequences (B). Individual bases were changed in the distal nVDRE sequence to create $5^{\prime}$ (5'MUT) and $3^{\prime}$ ( $3^{\prime}$ MUT) mutations, shown in lower case (C). Transcriptional activities of the mutant constructs in the full-length promoter as well as heterologous constructs were assessed by transient transfections in the ZR75-1 cells. (D) Functional activity of the mutations in the native promoter and (E) activity of the heterologous constructs with the various mutations. All plasmids were transiently transfected into the ZR75-1 cells followed by treatment with either a vehicle (Con) or $100 \mathrm{nM}$ calcitriol for $24 \mathrm{~h}$. Relative luciferase activity in the calcitriol-treated cells was compared with that of the vehicle-treated controls in each transfection that was set at $100 \%$. Values represent means \pm s.E.M. of at least four independent transfections performed in triplicate. $* * P<0.01$ and $* * * P<0.001$ when compared with the control. 


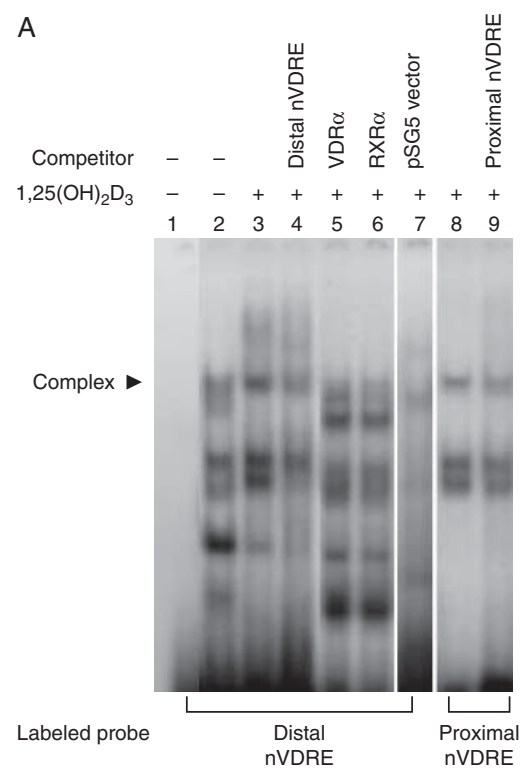

\section{Figure 4}

EMSA demonstrating the binding of the VDR to the ER nVDREs. (A) The nucleotides corresponding to the distal (lanes 1-7) and proximal (lanes 8 and 9) nVDREs were annealed and end-labeled with $\left[g^{32} \mathrm{P}\right]$ ATP using $T_{4}$ polynuleotide kinase. The labeled probes were then incubated with $5 \mu \mathrm{g}$ of extracts of ZR75-1 cells containing the PSG5-VDR expression vector in the absence (lanes 1 and 2) or presence (lanes 3-9) of calcitriol. Competition with $500 \times$ unlabeled probe can be seen in lanes 4 (distal nVDRE) and 9 (proximal nVDRE). Blockades by an anti-VDR antibody and an anti-RXR $\alpha$ antibody of the distal nVDRE binding are shown in lanes 5 and 6 . Lane 1 represents the free probe (no binding) and lane 7 represents the

weight complexes of unknown significance was detected (Fig. 4A, lanes 5 and 6). Lane 7 in Fig. 4A represents a control reaction using extracts from cells transfected with the pSG5 vector alone. The incubation of cell extracts with the radiolabeled proximal nVDRE probe led to the formation of a radiolabeled band, indicating protein-DNA complex formation (Fig. 4A, lane 8), and the band intensity was reduced upon competition with the unlabeled proximal nVDRE (Fig. 4A, lane 9).

Figure 4B depicts the competition exhibited by both WT and mutant nVDREs on complex formation. The intensity of the band representing the complex formed with a labeled distal nVDRE probe was reduced when competition was carried out with an unlabeled WT distal VDRE sequence (Fig. 4B, lane 4) but not with an unlabeled proximal VDRE sequence (Fig. 4B, lane 5) or unlabeled distal nVDRE $5^{\prime}$ or $3^{\prime}$ mutant sequences (Fig. 4B, lanes 6 and 7). When we used a radiolabeled distal nVDRE $5^{\prime}$ mutant sequence as the probe, the intensity of the complex formed (Fig. 4B, lane 8) was much weaker compared with that of the complex formed with the radiolabeled WT distal nVDRE (Fig. 4B, lane 3). In alternate
pSG5-empty vector (minimal binding). (B) Nucleotides corresponding to the WT distal nVDRE (lanes 1-7), the distal 5'MUT nVDRE (lanes 8 and 9), and the distal $3^{\prime}$ MUT nVDRE (lanes 10 and 11) were annealed and endlabeled with [ $\left.\mathrm{g}^{32} \mathrm{P}\right]$ ATP. Lane 1 represents the free WT distal nVDRE probe and lanes 2 and 3 represent those with and without calcitriol. Lanes 4-7 represent competition with various unlabeled probes as indicated. Specific binding of the VDR to the labeled 5'MUT and 3'MUT is shown in lanes 8-11. These mutations are the same as those introduced into the heterologous promoter constructs shown in Fig. 3.

experiments, when a radiolabeled distal nVDRE $3^{\prime}$ mutant sequence was used as the probe (Fig. 4B, lane 10), the complex formed had as much or more band intensity than that observed with the radiolabeled WT distal nVDRE probe (Fig. 4B, lane 3). These data suggest that the nucleotide bases in the $5^{\prime}$ hexameric half-site of the distal nVDRE are important for RXR-VDR heterodimer complexing with DNA. Competition exhibited by the unlabeled distal nVDRE $5^{\prime}$ and $3^{\prime}$ mutant oligonucleotides reduced the intensity of the complexes formed with their corresponding radiolabeled oligonucleotides (Fig. 4B, lanes 9 and 11).

\section{ChIP analysis of the binding of the VDR and RXR to the distal nVDRE}

To demonstrate that calcitriol recruits the VDR to the distal nVDRE in the native $E R$ promoter in intact cells, we carried out a ChIP analysis. ZR75-1 and MCF-7 cells exposed to $100 \mathrm{nM}$ calcitriol for $2 \mathrm{~h}$ were subjected to a ChIP assay. As shown in Fig. 5A, the ER promoter distal nVDRE region was co-immunoprecipitated with the anti-VDR (Fig. 5A, lanes 3 and 4) and anti-RXR $\alpha$ (Fig. 5A,

Published by Bioscientifica Ltd. 
A

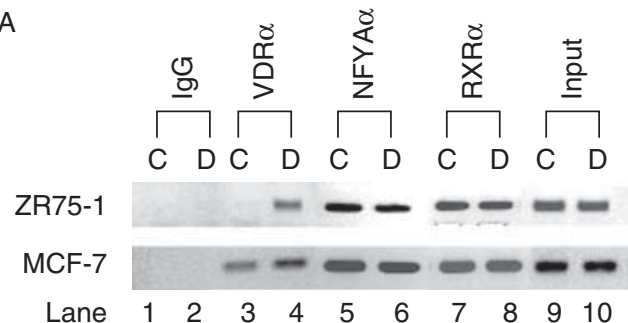

B

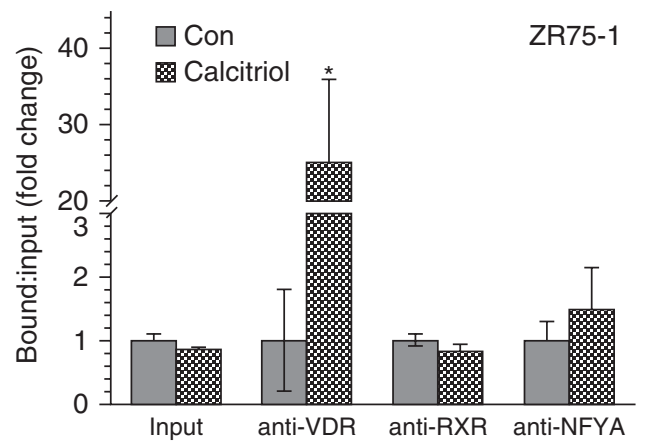

C

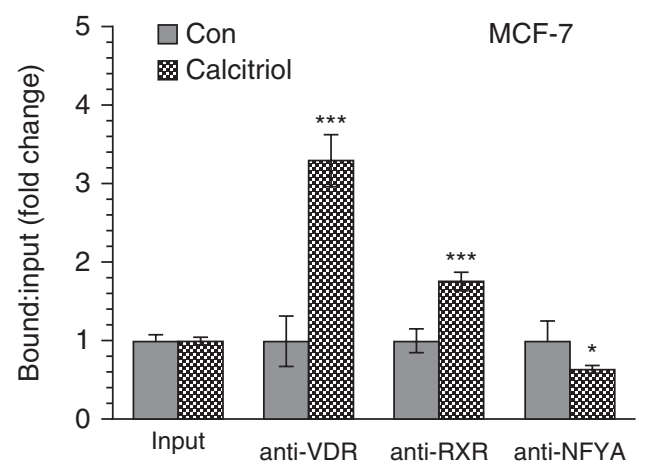

Figure 5

ChIP assay demonstrating the binding of the VDR, RXR, and NFYA to the distal nVDRE site in the ER promoter. (A) ZR75-1 and MCF-7 cells grown in $100 \mathrm{~mm}$ dishes were treated with a vehicle $(0.1 \%$ ethanol) or calcitriol for $2 \mathrm{~h}$ and subjected to a ChIP assay as described in the Subjects and methods section. After immunoprecipitation, the samples were analyzed by qPCR using primers designed to amplify a $178 \mathrm{bp} E R \alpha$ promoter fragment from -2581 to -2403 bp encompassing the distal nVDRE. Representative agarose gels: $C$, control, vehicle-treated cells and $D$, calcitriol $(100 \mathrm{nM})$-treated cells. Lanes 1 and 2 represent immunoprecipitation with nonspecific lgG, lanes 3 and 4 with a mixture of antiVDR antibodies, lanes 5 and 6 with an anti-NFYA antibody, and lanes 7 and 8 with an anti-RXR $\alpha$ antibody. Lanes 9 and 10 represent the input DNA. (B and C) DNA immunoprecipitated with the indicated antibody in the ZR75-1 (B) or MCF-7 (C) cells was analyzed by qPCR amplifying the distal $E R$ nVDRE region. The qPCR data are normalized to the amount of chromatin in the immunoprecipitation reaction (input). Data are represented as a ratio of the immunoprecipitated (bound) to input DNA. The bound:input ratio was set at 1 for vehicle treatment in each immunoprecipitation and was $0.14 \pm 0.07,4.4 \pm 0.6$, and $16.6 \pm 1.3$ respectively for VDR, NFYA, and RXR immunoprecipitations in the ZR75-1 Printed in Great Britain
D

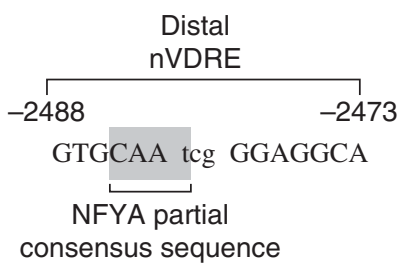

$E$

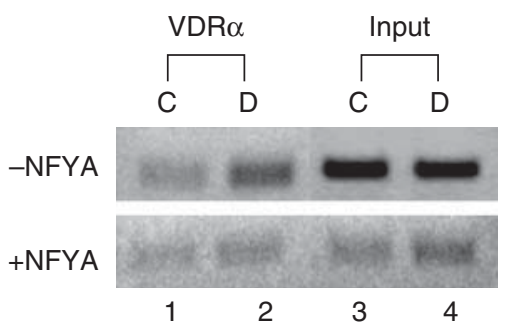

$\mathrm{F}$

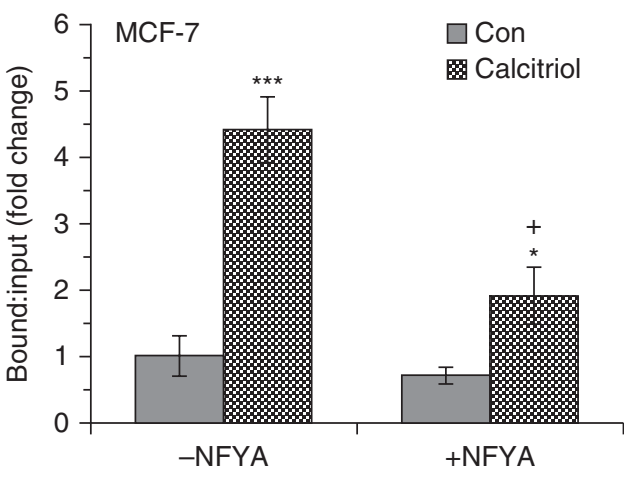

cells and $0.04 \pm 0.005,33.3 \pm 4.6$, and $11.5 \pm 1.2$ respectively for VDR, NFYA, and RXR in the MCF-7 cells. Values represent means \pm s.E.M. of three to six determinations. ${ }^{*} P<0.05$ and $* * * P<0.001$ when compared with the corresponding controls. (D) DNA sequence of the distal $E R$ nVDRE and its flanking regions. The shaded area demonstrates the presence of a partial NFY binding site that overlaps the distal nVDRE site in the ER promoter. (E and F) ChIP analysis of the binding of VDR to the distal $E R$ nVDRE site in the MCF-7 cells overexpressing NFYA. A NFYA expression plasmid (pSG5-NFYA) was transiently transfected into the MCF-7 cells. Untransfected (-NFYA) or NFYA-transfected (+NFYA) cells were treated with a vehicle (C) or $100 \mathrm{nM}$ calcitriol (D) for $2 \mathrm{~h}$, and a ChIP assay was carried out using a mixture of anti-VDR antibodies as described in the Subjects and methods section. (E) A representative agarose gel. (F) QPCR data of the ChIP analysis described in (E). The bound:input ratio was set at 1 for vehicle treatment in the VDR immunoprecipitation, which was $0.7 \pm 0.22$ in the untransfected ( - NFYA) cells and $0.6 \pm 0.09$ in the NFYA-transfected cells. Values represent means \pm S.E.M. of three determinations. $* P<0.05$ and $* * * P<0.001$ when compared with the corresponding controls. ${ }^{+} P<0.05$ compared with the calcitriol-treated + NFYA cells.

Published by Bioscientifica Ltd. 
lanes 7 and 8) antibodies, but not with IgG (Fig. 5A, lanes 1 and 2). In immunoprecipitation reactions with the antiVDR antibodies, significantly more PCR product was generated in cells treated with calcitriol (D) when compared with the vehicle control (C) (Fig. 5A, lanes 4 vs 3 ), indicating that calcitriol induced the recruitment of the VDR to the distal nVDRE sequence in the $E R$ promoter. Calcitriol induced an $\sim 25$-fold increase in the PCR product generated from DNA immunoprecipitated with the anti-VDR antibodies in the ZR75-1 cells (Fig. 5B) and an $\sim 3.5$-fold increase in the same in the MCF-7 cells (Fig. 5C). In contrast, calcitriol treatment did not affect the amount of PCR product generated from DNA immunoprecipitated with the anti-RXR $\alpha$ antibodies in the ZR75-1 cells (Fig. 5B), while a modest (approximately twofold) but statistically significant increase was observed in the MCF-7 cells (anti-RXR, Fig. 5C).

\section{Assessment of the role of NFY in the repression of $E R$ transcription by calcitriol}

We identified a putative binding site for nuclear factor $\mathrm{Y}$ (NFY), a transcription factor known to bind to the CCAAT box in the promoter regions of target genes to activate gene transcription (Kabe et al. 2005). As shown in Fig. 5D, a partial consensus NFY binding sequence $(-2486$ to $-2482 \mathrm{bp}$ ) is present within the sequence of the $15 \mathrm{mer}$ distal nVDRE ( -2488 to $-2473 \mathrm{bp}$ ). We, therefore, considered the possibility that calcitriol-mediated repression of $E R$ transcription is at least in part due to the competitive binding of the VDR and NFY to overlapping sites at the distal nVDRE locus. This would result in the displacement of NFY by the VDR, thereby attenuating the activation of $E R$ transcription by NFY. Alternatively, occupancy of the site by NFY would diminish the ability of calcitriol to repress $E R$ transcription by binding to the distal nVDRE site. To investigate this potential interaction, we carried our ChIP and transactivation assays.

Figure $5 \mathrm{~A}$ (lanes 5 and 6 ) shows that the $E R$ promoter distal nVDRE region was co-immunoprecipitated with an anti-NFYA antibody, suggesting the binding of endogenous levels of NFYA to this site. Calcitriol treatment did not affect the amount of PCR product generated from DNA immunoprecipitated with the anti-NFYA antibody in the ZR75-1 cells (Fig. 5B, anti-NFYA), while a small but statistically significant decrease was observed in the MCF-7 cells (Fig. 5C, anti-NFYA). In a separate experiment, a NFYA expression plasmid was transfected into the MCF-7 cells, following which a ChIP analysis was carried out using the anti-VDR antibodies in cells treated with a vehicle or calcitriol (Fig. 5E and F).

In the absence of NFYA overexpression with only endogenous NFYA levels (Fig. 5E, - NFYA), calcitriol induced an approximately fourfold increase in the amount of PCR product generated from the immunoprecipitated DNA (Fig. 5F, - NFYA). However, NFYA overexpression (Fig. 5E, + NFYA) significantly reduced the calcitriol-induced increase in the PCR product generated from DNA immunoprecipitated with the antiVDR antibodies (Fig. 5F, + NFYA, $P<0.05$ ), suggesting an interference of the ligand-induced recruitment of the VDR to the distal nVDRE site by NFYA.

In transactivation assays, we transiently transfected the NFYA expression plasmid along with pSG5-VDR and the full-length $E R$ promoter-reporter construct $(-3500$ to +230 ER promoter-luc) into the ZR75-1 and MCF-7 cells and measured reporter activity following calcitriol treatment. As expected, calcitriol treatment resulted in a repression of reporter activity $(\sim 55 \%)$ in the vectortransfected ZR75-1 cells (Fig. 6A). Co-transfection of the WT NFYA expression plasmid did not stimulate the basal promoter activity. However, NFYA co-expression greatly reduced the calcitriol-mediated suppression of $E R$ promoter activity. However, a significant calcitriol-mediated suppression of reporter activity still remained when a mutant form of NFYA was co-expressed (Fig. 6A). Similar results were observed when the effect of NFYA overexpression on $E R$ promoter activity was examined in the MCF-7 cells (Fig. 6B). We also carried out western blot analysis to determine the changes in $E R$ protein levels due to calcitriol treatment in the ZR75-1 cells (Fig. 6C) and MCF-7 cells (Fig. 6D) transfected with vector, WT NFYA, and mutant NFYA expression plasmids. The data were similar to the changes observed in $E R$ promoter activity. Co-expression of the WT NFYA but not of the mutant NFYA or vector abolished the calcitriolinduced suppression of $E R$ protein levels (Fig. 6C and 6D). These data indicate that although NFYA by itself did not act as a positive regulator of $E R$ transcription in this experimental setting, its co-expression resulted in the loss of the suppression of $E R$ gene expression by calcitriol, suggesting that it probably competes for binding to the distal nVDRE.

\section{Discussion}

The factors and mechanisms that control ER levels are important in determining the magnitude of estrogenic actions in BCa cells. Studies conducted in our laboratory (Swami et al. 2000) and other laboratories (James et al. 1994, Simboli-Campbell et al. 1997, Stoica et al. 1999)

Published by Bioscientifica Ltd. 
A

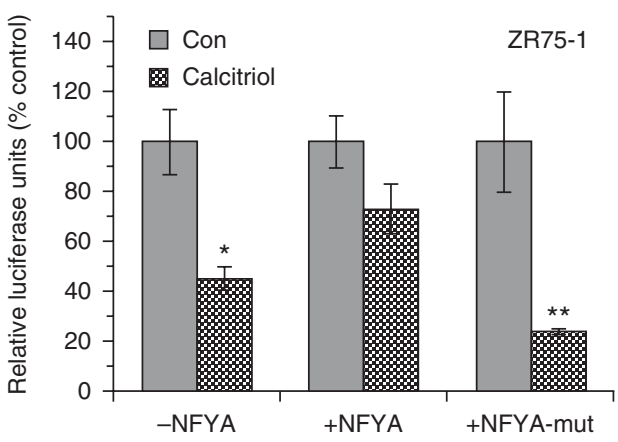

C

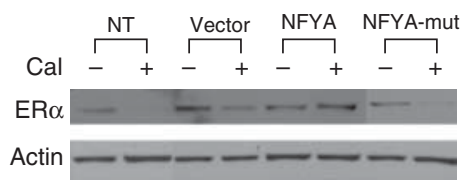

\section{Figure 6}

Interference of NFY with calcitriol suppression of $E R$ promoter activity and ER expression. ZR75-1 (A) and MCF-7 (B) cells were transiently transfected with a WT ER promoter construct $(\sim 3.5 \mathrm{bp})$ in the absence or presence of pSG5-NFYA or pSG5-NFYA-mut plasmids as described in the Subjects and methods section. The cells were treated with a vehicle (Con) or calcitriol $(100 \mathrm{nM})$ for $24 \mathrm{~h}$ and luciferase activity was measured. Relative luciferase activity in the calcitriol-treated cells was compared with that of the vehicletreated controls in each transfection that was set at $100 \%$. Data represent means \pm S.E.M. of at least four independent transfections, performed in triplicate. ${ }^{*} P<0.05,{ }^{*} P<0.01$ and ${ }^{* * *} P<0.001$ when compared with their

have demonstrated that calcitriol downregulates $E R$ expression in human BCa cells. We have shown that by decreasing ER levels, calcitriol causes a significant attenuation of estrogen-mediated bioresponses in MCF-7 cells, including reduction of estrogen stimulation of cell growth (Swami et al. 2000). The mechanism of calcitriol regulation of $E R$ expression appears to be a direct repression of $E R$ transcription by calcitriol (Stoica et al. 1999, Swami et al. 2000). In this study, we have further analyzed the mechanism of the transrepression of the $E R$ gene by calcitriol.

The transcriptional regulation of target genes is accomplished by the binding of calcitriol binding to the VDR, which after heterodimerization with RXR binds to specific VDREs located in the promoter regions of target genes as well as to distant enhancers. VDREs typically consist of a direct repeat (DR) of two hexameric core binding motifs separated by a spacer most often consisting of three (DR3) and sometimes four (DR4) nucleotides (Carlberg 2003, Haussler et al. 2011, Pike et al. 2011b). The VDR has also been shown to bind to some unconventional VDREs such as the DR-type VDRE with a

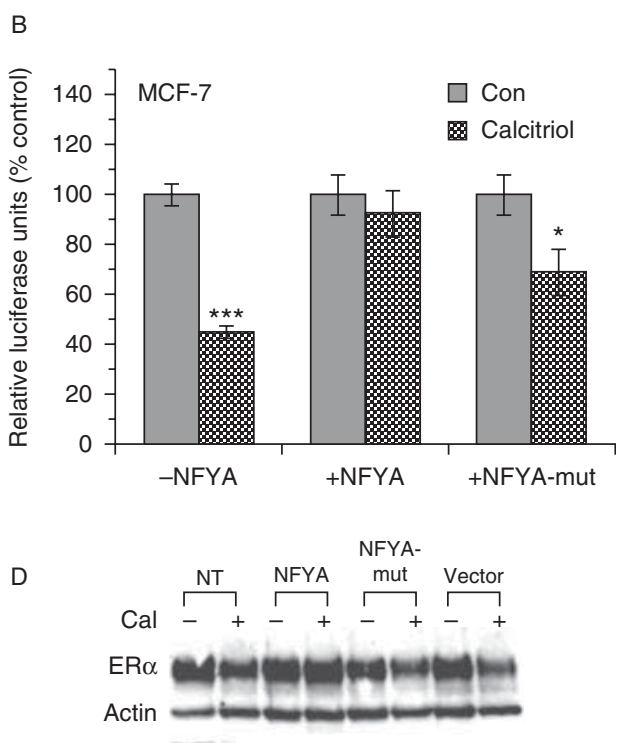

respective controls. (C) ZR75-1 cells and (D) MCF-7 cells are representative western blots showing $E R$ expression in the presence or absence of NFYA overexpression. Briefly, cells transiently transfected with the pSG5-NFYA or pSG5-NFYA-mut expression vectors were treated with a vehicle or calcitriol $(100 \mathrm{nM})$ for a period of $48 \mathrm{~h}$ after transfection. Empty vector-transfected (Vector) cells and non-transfected (NT) cells were similarly treated and used as negative controls in the experiment. At the end of treatment, the cells were processed for western blot analysis as described in the Subjects and methods section.

larger spacer or an inverted palindromic arrangement (Carlberg 2003). In the case of transcriptional repression, however, direct VDR DNA binding or formation of a heterodimer with RXR may not always be required and the mechanism of repression may be target gene specific. For example, the repression of 25-hydroxyvitamin D-1 $\alpha$ hydroxylase (CYP27B1) gene transcription in the kidney by calcitriol involves the inhibition by the VDR of the actions of the transcription factor vitamin $\mathrm{D}$ interacting repressor, which is essential for regulating the basal CYP27B1 expression (Murayama et al. 2004). CYP27B1 repression has also been shown to involve calcitriolinduced epigenetic changes such as DNA methylation (Kim et al. 2007).

The current study and that of Stoica et al. (1999) have demonstrated the presence and functionality of two nVDREs in an $\sim 3.5 \mathrm{~kb}$ promoter region of the $E R$ gene. Recent genome-wide approaches have revealed that calcitriol regulation of target genes can be orchestrated not only by multiple regulatory regions located proximal to the promoters but often by many distal enhancers located several hundreds of kilobases away in either

Published by Bioscientifica Ltd 
intergenic regions surrounding the transcription units or within the introns themselves (Pike \& Meyer 2010, Pike 2011, Pike et al. 2011a). However, the current study shows that together the functional activities of the two nVDRE sites within the $\sim 3.5 \mathrm{~kb} E R$ promoter region recapitulate the magnitude of the downregulation of $E R$ expression observed in BCa cells following calcitriol treatment. These VDRE sites are referred to as the proximal nVDRE located at -94 to $-70 \mathrm{bp}$ with reference to the P1 start site and the distal nVDRE further upstream at -2488 to $-2473 \mathrm{bp}$ in the $E R$ promoter. The proximal nVDRE, identified and characterized by Stoica et al. (1999), is an imperfect palindrome separated by 13 nucleotides, which maps in the region of a CTF/NF1 site in the $E R$ promoter. The current study reports the presence and analysis of another upstream nVDRE (distal). The functionality of these nVDREs is strongly supported by the fact that these sequences confer calcitriol responsiveness both in the natural $E R$ promoter and in a heterologous promoter setting. The individual proximal and distal nVDRE sites appear to contribute equally to the negative regulation of the $E R$ gene by calcitriol as demonstrated by the fact that a heterologous construct containing both the nVDRE sites exhibited an $\sim 50 \%$ decrease in reporter activity, recapitulating the magnitude of suppression reported by the measurement of ER mRNA and protein levels in BCa cells (Swami et al. 2000). The specific binding of the VDR to these nVDRE sites was demonstrated by EMSA. The addition of the anti-VDR and anti-RXR antibodies revealed the inhibition of protein-DNA complex formation in EMSA at the distal nVDRE site. Furthermore, the results of the ChIP assay demonstrated that calcitriol recruits the VDR to the distal nVDRE site in the context of the native $E R$ promoter in $\mathrm{BCa}$ cells and that $\mathrm{RXR} \alpha$ is also bound to this site most probably as a heterodimer with the VDR. Mutations in the $5^{\prime}$ half-site but not in the $3^{\prime}$ half-site of the distal nVDRE abolished the suppressive effect of calcitriol in transactivation assays and inhibited complex formation in the EMSA, suggesting that the $5^{\prime}$ hexameric sequence is required for calcitriol suppressor activity via the distal nVDRE.

Competition for binding with factors that stimulate transcription has been implicated in the repressive effects of calcitriol on the promoters of several target genes such as rat bone sialoprotein (Kim et al. 1996), runt-related transcription factor 2 (RUNX2/Cbfa1) (Drissi et al. 2002), interleukin 2 (Alroy et al. 1995), and human parathyroid hormone (hPTH; Koszewski et al. 2004, Jaaskelainen et al. 2005). The CCAAT box is one of the most typical elements for transcriptional activation. NFY is one among the different proteins that bind to CCAAT sequences on many promoter regions and recruit RNA polymerase II to these promoters (Kabe et al. 2005). Recently, NFY has been implicated in the positive regulation of $\mathrm{p} 27^{\mathrm{Kip} 1}$ promoter by calcitriol (Inoue et al. 1999, Huang et al. 2004) as well as in the suppression of the $\mathrm{h} P T H$ promoter by calcitriol (Koszewski et al. 2004, Jaaskelainen et al. 2005). Two consensus NFY binding sites have been identified in the $\mathrm{h} P T H$ promoter, and the repressive effect of calcitriol on $\mathrm{h} P T H$ gene transcription may involve the displacement of NFY binding to its proximal site by the VDR-RXR heterodimer (Koszewski et al. 2004). Cooperation between NFY bound to both the distal and proximal consensus sites causes synergistic transactivation of $\mathrm{hPTH}$ and calcitriol appears to attenuate this effect by disrupting NFY binding to its proximal consensus site (Koszewski et al. 2004). We have found a partial NFY binding site (CAAT) at -2486 to $-2482 \mathrm{bp}$ present within the sequences of the distal nVDRE on the ER promoter ( -2488 to $-2473 \mathrm{bp}$ ). This finding prompted us to investigate the possibility that the calcitriol-mediated repression of $E R$ transcription is at least in part due to the competitive binding of the VDR to the NFY site and thereby attenuates the transcriptional activity of NFY.

Our analysis of the role of NFY in calcitriolmediated transrepression of $E R$ revealed several interesting findings. Our ChIP data demonstrate that endogenous levels of NFY bind to the distal nVDRE site in both ZR75-1 and MCF-7 cells. Although the data obtained from the MCF-7 cells suggest that calcitriol treatment, probably through the recruitment of the VDR to the distal nVDRE site, reduces endogenous NFY binding to this site, we did not detect this in the ZR75-1 cells. NFY overexpression in the MCF-7 cells clearly reduced calcitriol-induced VDR recruitment to this site. In transactivation assays, unlike the case of the $\mathrm{h} P T H$ promoter, the activity of which was robustly stimulated by NFY expression, we did not find a stimulation of $E R$ promoter activity even when NFYA was overexpressed in either the ZR75-1 or MCF-7 cells. However, the overexpression of the WT NFYA but not the mutant NFYA resulted in the loss of calcitriol-mediated suppression of $E R$ promoter activity and of $E R$ protein expression in these cells. NFY has a stringent requirement for the CCAAT sequence and its flanking nucleotides for efficient DNA binding. A majority of the NFY binding sequences have the intact CCAAT core. However, in the case of a few NFY binding sites with a one-nucleotide difference in the core, a much lower DNA binding affinity of NFY has been reported

Published by Bioscientifica Ltd 
(Mantovani 1999). It is possible that the lack of the intact CCAAT core sequence within the distal nVDRE might have contributed to less-than-optimal binding of NFY to the distal nVDRE site and the absence of a positive stimulation of $E R$ transcription by NFY. It is also possible that other NFY binding sites that might be present further upstream were lacking in the $E R$ promoter constructs that we tested, contributing to the absence of stimulation of the basal promoter activity by NFY. Our data suggest that even though we did not observe positive regulator activity, NFYA did bind to its putative binding site present within the distal nVDRE and inhibited the recruitment of the VDR complex to the distal nVDRE site, thereby reducing the repressive effect of calcitriol on $E R$ transcription. It is interesting to note that as reported by Stoica et al. (1999) the proximal nVDRE also overlaps a potential binding site for the CCAAT box binding transcriptional stimulator CTF/NF1 . However, it should be emphasized that when either the distal or proximal nVDRE was placed upstream of the SV40 promoter in a heterologous construct, it still acted independently as a negative response element, indicating that $E R$ repression by calcitriol may not be solely due to interference with the stimulatory activity of positive factors such as NFY and CTF/NF1.

Calcitriol inhibits the proliferation of $\mathrm{BCa}$ cells through a variety of mechanisms and suppresses tumor growth in animal models of BCa (Deeb et al. 2007, Krishnan \& Feldman 2011, Welsh 2011). We have shown that calcitriol acts as a SAM, decreasing aromatase expression in $\mathrm{BCa}$ cells and the breast adipose tissue surrounding a breast tumor, thereby reducing the local production of estrogens within the breast (Krishnan et al. $2010 b$, Swami et al. 2011, 2012). In addition to suppressing estrogen synthesis, the current study demonstrates that calcitriol also transrepresses $E R$ through the nVDRE elements present in the $E R$ promoter, leading to a decrease in the magnitude of estrogenic bioresponses (Swami et al. 2000). The combined actions of calcitriol to inhibit both estrogen synthesis and estrogen signaling through the ER lead to the diminution of the proliferative stimulus for $\mathrm{BCa}$ growth provided by estrogens. These observations raise the possibility that calcitriol may be useful in the prevention and/or treatment of $\mathrm{ER}+\mathrm{BCa}$.

\section{Declaration of interest}

The authors declare that there is no conflict of interest that could be perceived as prejudicing the impartiality of the research reported.
Funding

This work was supported by NCl Grant (CA13099) and Komen Foundation Grant (070101) to D Feldman.

\section{Acknowledgements}

We thank Dr Ronald Weigel for the kind gift of $E R$ promoter deletion plasmids and Dr Roberto Mantovani for the kind gift of the NFYA expression plasmids.

\section{References}

Alroy I, Towers TL \& Freedman LP 1995 Transcriptional repression of the interleukin-2 gene by vitamin D3: direct inhibition of NFATp/AP-1 complex formation by a nuclear hormone receptor. Molecular and Cellular Biology 15 5789-5799.

Beer TM \& Myrthue A 2004 Calcitriol in cancer treatment: from the lab to the clinic. Molecular Cancer Therapeutics 3 373-381.

Burstein HJ, Prestrud AA, Seidenfeld J, Anderson H, Buchholz TA, Davidson NE, Gelmon KE, Giordano SH, Hudis CA, Malin J et al. 2010 American Society of Clinical Oncology clinical practice guideline: update on adjuvant endocrine therapy for women with hormone receptor-positive breast cancer. Journal of Clinical Oncology 28 3784-3796. (doi:10.1200/JCO.2009.26.3756)

Carlberg C 2003 Molecular basis of the selective activity of vitamin D analogues. Journal of Cellular Biochemistry 88 274-281. (doi:10.1002/ jcb.10337)

deConinck EC, McPherson LA \& Weigel RJ 1995 Transcriptional regulation of estrogen receptor in breast carcinomas. Molecular and Cellular Biology 15 2191-2196.

Deeb KK, Trump DL \& Johnson CS 2007 Vitamin D signalling pathways in cancer: potential for anticancer therapeutics. Nature Reviews. Cancer $\mathbf{7}$ 684-700. (doi:10.1038/nrc2196)

Demirpence E, Balaguer P, Trousse F, Nicolas JC, Pons M \& Gagne D 1994 Antiestrogenic effects of all-trans-retinoic acid and 1,25-dihydroxyvitamin D3 in breast cancer cells occur at the estrogen response element level but through different molecular mechanisms. Cancer Research 54 1458-1464.

Drissi H, Pouliot A, Koolloos C, Stein JL, Lian JB, Stein GS \& van Wijnen AJ 2002 1,25-(OH)2-vitamin D3 suppresses the bone-related Runx2/Cbfa1 gene promoter. Experimental Cell Research 274 323-333. (doi:10.1006/ excr.2002.5474)

Feldman D, Krishnan AV \& Swami S 2013 Vitamin D: biology, action and clinical implications. In Osteoporosis, 4th edn, vol 1, pp 283-329. Eds R Marcus, D Feldman, D Dempster, M Luckey \& J Cauley. San Diego: Elsevier Academic Press.

Haussler MR, Whitfield GK, Haussler CA, Hsieh J-C \& Jurutka PW 2011 Nuclear vitamin D receptor: natural ligands, molecular structurefunction and transcriptional control of vital genes. In Vitamin D, pp 137-170. Eds D Feldman, JW Pike \& JS Adams., 3rd edn San Diego: Elsevier Academic Press.

Huang YC, Chen JY \& Hung WC 2004 Vitamin D3 receptor/Sp1 complex is required for the induction of p27Kip1 expression by vitamin D3. Oncogene 23 4856-4861. (doi:10.1038/sj.onc.1207621)

Inoue T, Kamiyama J \& Sakai T 1999 Sp1 and NF-Y synergistically mediate the effect of vitamin $\mathrm{D}(3)$ in the $\mathrm{p} 27$ (Kip1) gene promoter that lacks vitamin D response elements. Journal of Biological Chemistry 274 32309-32317. (doi:10.1074/jbc.274.45.32309)

Jaaskelainen T, Huhtakangas J \& Maenpaa PH 2005 Negative regulation of human parathyroid hormone gene promoter by vitamin D3 through nuclear factor Y. Biochemical and Biophysical Research Communications 328 831-837. (doi:10.1016/j.bbrc.2005.01.033) 
James SY, Mackay AG, Binderup L \& Colston KW 1994 Effects of a new synthetic vitamin D analogue, EB1089, on the oestrogen-responsive growth of human breast cancer cells. Journal of Endocrinology $\mathbf{1 4 1}$ 555-563. (doi:10.1677/joe.0.1410555)

Kabe Y, Yamada J, Uga H, Yamaguchi Y, Wada T \& Handa H 2005 NF-Y is essential for the recruitment of RNA polymerase II and inducible transcription of several CCAAT box-containing genes. Molecular and Cellular Biology 25 512-522. (doi:10.1128/MCB.25.1.512-522.2005)

Kim RH, Li JJ, Ogata Y, Yamauchi M, Freedman LP \& Sodek J 1996 Identification of a vitamin D3-response element that overlaps a unique inverted TATA box in the rat bone sialoprotein gene. Biochemical Journal 318 219-226.

Kim MS, Fujiki R, Kitagawa H \& Kato S 2007 1 $\alpha, 25(\mathrm{OH}) 2 \mathrm{D} 3$-induced DNA methylation suppresses the human CYP27B1 gene. Molecular and Cellular Endocrinology 265-266 168-173. (doi:10.1016/j.mce.2006. 12.014)

Koszewski NJ, Alimov AP, Park-Sarge OK \& Malluche HH 2004 Suppression of the human parathyroid hormone promoter by vitamin $\mathrm{D}$ involves displacement of NF-Y binding to the vitamin D response element. Journal of Biological Chemistry 279 42431-42437. (doi:10.1074/jbc. M407742200)

Krishnan AV \& Feldman D 2011 Mechanisms of the anti-cancer and antiinflammatory actions of vitamin D. Annual Review of Pharmacology and Toxicology 51 311-336. (doi:10.1146/annurev-pharmtox-010510100611)

Krishnan AV, Swami S \& Feldman D 2010a Vitamin D and breast cancer: inhibition of estrogen synthesis and signaling. Journal of Steroid Biochemistry and Molecular Biology 121 343-348. (doi:10.1016/j.jsbmb. 2010.02.009)

Krishnan AV, Swami S, Peng L, Wang J, Moreno J \& Feldman D $2010 b$ Tissue-selective regulation of aromatase expression by calcitriol: implications for breast cancer therapy. Endocrinology 151 32-42. (doi:10.1210/en.2009-0855)

Mantovani R 1999 The molecular biology of the CCAAT-binding factor NF-Y. Gene 239 15-27. (doi:10.1016/S0378-1119(99)00368-6)

McDonnell DP \& Wardell SE 2010 The molecular mechanisms underlying the pharmacological actions of ER modulators: implications for new drug discovery in breast cancer. Current Opinion in Pharmacology 10 620-628. (doi:10.1016/j.coph.2010.09.007)

Murayama A, Kim MS, Yanagisawa J, Takeyama K \& Kato S 2004 Transrepression by a liganded nuclear receptor via a bHLH activator through co-regulator switching. EMBO Journal 23 1598-1608. (doi:10.1038/sj.emboj.7600157)

Nilsson S, Koehler KF \& Gustafsson JA 2011 Development of subtypeselective oestrogen receptor-based therapeutics. Nature Reviews. Drug Discovery 10 778-792. (doi:10.1038/nrd3551)

Peng L, Malloy PJ \& Feldman D 2004 Identification of a functional vitamin D response element in the human insulin-like growth factor binding protein-3 promoter. Molecular Endocrinology 18 1109-1119. (doi:10.1210/me.2003-0344)
Pereira F, Larriba MJ \& Munoz A 2012 Vitamin D and colon cancer. Endocrine-Related Cancer 19 R51-R71. (doi:10.1530/ERC-11-0388)

Pike JW 2011 Genome-wide principles of gene regulation by the vitamin D receptor and its activating ligand. Molecular and Cellular Endocrinology 347 3-10. (doi:10.1016/j.mce.2011.05.012)

Pike JW \& Meyer MB 2010 The vitamin D receptor: new paradigms for the regulation of gene expression by 1,25-dihydroxyvitamin $\mathrm{D}(3)$. Endocrinology and Metabolism Clinics of North America 39 255-269. (doi:10.1016/j.ecl.2010.02.007)

Pike JW, Meyer MB \& Bishop KA 2011a Regulation of target gene expression by the vitamin D receptor - an update on mechanisms. Reviews in Endocrine \& Metabolic Disorders 13 37-51.

Pike JW, Meyer MB \& Lee SM 2011 $b$ The vitamin D receptor: biochemical, molecular, biological and genomic era investigations. In Vitamin $D$, pp 97-135. Eds D Feldman, JW Pike \& JS Adams., 3rd edn San Diego: Elsevier Academic Press.

Siegel R, Naishadham D \& Jemal A 2012 Cancer statistics, 2012. CA: A Cancer Journal for Clinicians 62 10-29. (doi:10.3322/caac.20138)

Simboli-Campbell M, Narvaez CJ, van Weelden K, Tenniswood M \& Welsh J 1997 Comparative effects of 1,25(OH)2D3 and EB1089 on cell cycle kinetics and apoptosis in MCF-7 breast cancer cells. Breast Cancer Research and Treatment 42 31-41. (doi:10.1023/A:1005772432465)

Stoica A, Saceda M, Fakhro A, Solomon HB, Fenster BD \& Martin MB 1999 Regulation of estrogen receptor- $\alpha$ gene expression by 1, 25-dihydroxyvitamin D in MCF-7 cells. Journal of Cellular Biochemistry 75 640-651. (doi:10.1002/(SICI)1097-4644(19991215)75:4<640::AID-JCB10>3.0. CO;2-8)

Swami S, Krishnan AV \& Feldman D 2000 1 1,25-Dihydroxyvitamin D3 down-regulates estrogen receptor abundance and suppresses estrogen actions in MCF-7 human breast cancer cells. Clinical Cancer Research 6 3371-3379.

Swami S, Krishnan AV, Wang JY, Jensen K, Peng L, Albertelli MA \& Feldman D 2011 Inhibitory effects of calcitriol on the growth of MCF-7 breast cancer xenografts in nude mice: selective modulation of aromatase expression in vivo. Hormones \& Cancer 2 190-202. (doi:10.1007/s12672-011-0073-7)

Swami S, Krishnan AV, Wang JY, Jensen K, Horst R, Albertelli MA \& Feldman D 2012 Dietary vitamin D3 and 1,25-dihydroxyvitamin D3 (calcitriol) exhibit equivalent anticancer activity in mouse xenograft models of breast and prostate cancer. Endocrinology 153 2576-2587. (doi:10.1210/en.2011-1600)

Tomao F, Spinelli G, Vici P, Pisanelli GC, Cascialli G, Frati L, Panici PB \& Tomao S 2011 Current role and safety profile of aromatase inhibitors in early breast cancer. Expert Review of Anticancer Therapy 11 1253-1263. (doi:10.1586/era.11.96)

Welsh J 2011 Vitamin D metabolism in mammary gland and breast cancer. Molecular and Cellular Endocrinology 347 55-60. (doi:10.1016/j.mce. 2011.05.020)

Welsh J 2012 Cellular and molecular effects of vitamin D on carcinogenesis. Archives of Biochemistry and Biophysics 523 107-114. (doi:10.1016/j.abb. 2011.10.019)

Received in final form 29 April 2013

Accepted 4 June 2013

Made available online as an Accepted Preprint

6 June 2013 http://erc.endocrinology-journals.org DOI: 10.1530/ERC-12-0281
(C) 2013 Society for Endocrinology Printed in Great Britain
Published by Bioscientifica Ltd. 